\title{
ESSAI SUR LES FORMES GÉOMÉTRIQUES DES COLONIES DE CERTAINS CHAMPIGNONS PATHOGÈNES
}

\author{
Par BERTHOLD WEISSBERG
}

On est frappé par la forme régulière, géométrique, des colonies d'un grand nombre de champignons. Il s'agit de chercher une explication pour cette régularité, sa constance sous des conditions identiques et sa variabilité avec les changements des conditions extérieures. Rappelons d'abord quelques exemples de ces colonies géométriques.

Dans un premier groupe, la colonie est divisée en plusieurs secteurs par des plis ou des sillons radiaires. Ainsi, la colonie de Microsporon audouini se présente sous l'aspect d'un gâteau plat,

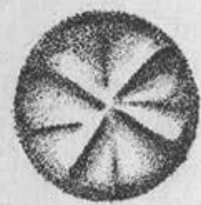

A

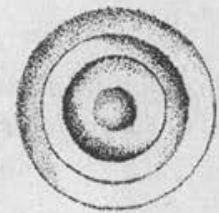

B

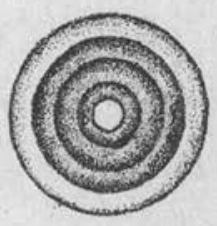

C

Fig. 1. - Colonies divisées en secteurs par des plis ou des sillons radiaires :

A, Microsporum audouini ; des sillons circulaires : B, Trichophyton tonsurans;

C, Microsporum circulus centrum.

silionné par des plis rayonnants qui partent d'une petite saillie centrale (fig. 1, A).

Un deuxième groupe se caractérise par la présence de plis, de sillons ou des anneaux circulaires, concentriques. Exemple : les colonies de Trichophyton tonsurans ou de Microsporon circuluscentrum (fig. $1, \mathrm{~B}$ et $\mathrm{C}$ ).

Dans un troisième groupe, enfin, les colonies ont des formes un peu plus compliquées. Ainsi, la colonie de Trichophyton polygonum est ronde et cratériforme au début, pour devenir ensuite polygonale et pour finir le plus souvent comme tétragone plus ou moins régulier. Autres exemples: Trichophyton rosaceum, dont les colonies

Ann. de Parasitologie, T. XXII, Nos 3-4, 1947, p. 242-253. 
revêtent des formes en rosette, ou Hemispora stellata, qui forme dans la nature des disques couverts de conidiophores, dessinant à leur surface des étoiles ou des marguerites (fig. 2, D, E, F).

Enfin, dernier exemple, la culture de Ctenomyces persicolor est d'abord ronde, et devient ensuite polygonale et étoilée, divisée en secteurs plus ou moins réguliers par des incisures radiaires (fig. 3 ).

On trouve ci-joint une liste des principaux champignons pathogènes à colonies plus ou moins régulières. Un certain nombre de culonies cérébriformes a été inclus dans cette liste, car on a souvent l'impression que cette plicature cérébriforme pourrait être quelque chose de secondaire, dû à un manque de place pour un épanouissement plus régulier des hyphes.

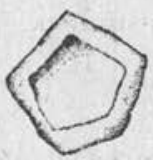

D

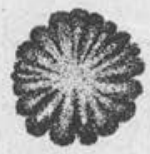

E

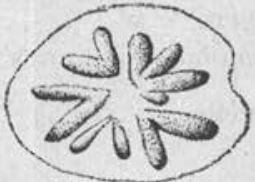

F

Fig. 2. - Colonies polygonales ou en rosette: D, Trichophyton polygonum ; E, T. rosaceum ; F, Hemispora stellata.

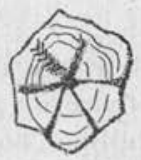

FIG. 3. - Ctenomycẹs persicolor : colonie polygonale, divisée en secteurs par des incisures radiaires.

\section{HYPHOMYCÈTES}

I. Microsiphonés :

Sreptomyces : striation radiaire; $S$. decussatus : colonies en forme de petites croix.

II. Thallosporés :

Arthrosporés.

Geotrichoïdes : G. rabesalama : colonies étoilées ; G. versiformis : colonies rayonnantes ou vermiculées.

Trichosporon : $T$. asteroides : colonies rayonnantes, cratériformes à la fin.

Blastosporés.

Candida : C. albicans : parfois sillons peu profonds. 


\section{Hémisporés :}

Hemispora : H. stellata : dans la nature, le mycelium forme des disques de 0,2 à $2,5 \mathrm{~mm}$. de diamètre, sessiles, couverts de conidiophores dessinant à leur surface des étoiles ou des marguerites en relief.

IV. Conidiosporés :

Cephalosporium : C. kiliense : colonies rayonnées ; C. stümeri : faisceaux de filaments et quelques sillons profonds, rayonnants.

Cladosporium : C. penicilliodes : cérébriformes.

Hormodendron : $H$. langeroni : sillons rayonnants et circulaires.

Malbranchea : M. kambayashii : cratériforme, sillonnée à la périphérie.

\section{Ascomycètes}

I. Hémiascomycètes :

Debaryomyces.

D. guilliermondi : colonies rondes à bords semi-sinueux.

II. Plectomycètes :

\section{Achorion :}

A. formoseum : partie aérienne plissée, partie végétant dans le milieu, émet des prolongement radiés.

A. schönleini : ressemble à la morille.

A. schönleini : var. mongolica : disque à contour frangé, émettant quelques rayons irréguliers.

\section{Ctenomyces :}

C. denticulatus : tapis rond frangé de denticulations.

C. eriotrephon : cratère entouré de plis rayonnants.

C. farinulentus : disque ombiliqué, coupé de radiations plus ou moins creuses. Souvent à la périphérie, auréole de rayons immergés.

Sur gélose peptonée sans sucre : d'abord coupole aplatie, puis découpée à la périphérie par des rayons qui se multiplient si la colonie vieillit.

C. granulosus : disque avec ombilic radié au centre ; surface granuleuse. Sur peptone à $30 / 0$, pourtour denticulé.

C. lacticolor: disque avec des cannelures radiées, parfois plus irrégulier.

C. mentagrophytes : centre entouré d'une auréole. Sillons rayonnants. Périphérie : nombreuses prolongations fines.

C. persicolor : rond, puis polygonal et étoilé, divisé en secteurs plus ou moins réguliers par des incisures radiées. Sur milieu peptoné : bords déchiquetés, élégants et à surface marquée de cercles concentriques.

C. radians : ombiliqué au centre, entouré à la périphérie par un grand nombre de fins rayons ondulés.

C. radiolatus : ressemble beaucoup à $C$. mentagrophytes.

C. radioplicatus : plis rayonnants, n'atteignant pas la périphérie. 


\section{Microsporum :}

M. audouini : disque sillonné par des plis radiaires.

M. circuluscentrum : centre blanc entouré de cercles de différentes couleurs.

M. equinum : colonie radiée de plis profonds qui, au centre, se contournent et se chiffonnent. Sur moût de bière gélosé, plicatures godronnées très régulières.

M. lanosum : aire centrale entourée d'un anneau de duvet blanc en relief. Pourtour à rayons immergés.

M. pubescens : umbo central, puis petite aire divisée par des cannelures en 3 ou 4 secteurs. En dehors, large anneau de duvet.

M. tomentosum: ombilic polygonal ourlé, au centre un petit umbo. Surface partagée en secteurs par des fossettes radiées.

$M$. umbonatum: forme ronde du bouclier antique, partagé en secteurs par des fossettes rayonnées (forme florale !).

$M$. villosum : disque de 6 centimètres, dont le centre plat montre l'ébauche de sillons radiés. Autour du centre, couronne de petits mamelons, entourée de sillons immergés.

\section{Trichophyton :}

T. effractum : ressemble à une fleur.

T. exsiccatum : cratère à large liseré, coupé de plis radiaires.

$T$. fuscum et $T$. sulcatum : d'abord cratériformes, s'affaissent ensuite et présentent des sillons rayonnants, anguleux, à angles droits ou aigus. Plus tard, frange homogène, non sillonnée.

T. polygonum: d'abord cratériforme, devient polygonal. Presque toujours quadrilatère. Surface chiffonnée à gros plis.

T. regulare : «On dirait une roue, dont le moyeu aurait été enlevé. »

T. sabouraudi : cône aplati, découpé en secteurs par des cannelures radiées.

T. tonsurans : le centre se creuse en capsule, les bords se relèvent.

$T$. langeroni : rayons épais, immergés, ramifiés, et masse centrale entourée d'îlots formés par les portions émergées des rayons.

$T$. areolatum : d'abord comme $T$. sabouraudi, à la fin cocarde.

T. gourvili : couvert de plis et de circonvolutions.

$T$. lanoroseum : pigment en anneaux.

T. multicolor : cérébriforme, polychrome.

$T$. plurizoniforme : surface très radiée, marquée de zones pigmentées.

T. rubrum : cannelures radiées.

$T$. violaceum : sillons ridés.

$T$. flavum (cerebriforme) : ressemble assez à $T$. tonsurans. Rayons poudreux dans la périphérie, aspect cérébriforme.

T. plicatile : périphérie moins rayonnée.

$T$. megnini (rosaceum) : secteurs qui ont tendance à s'arrondir.

$T$. vinosum : umbo au centre, puis radié, ourlé à la périphérie. 
T. album : zone centrale faviforme, auréole poudreuse blanche et auréole incolore de rayons immergés.

Penicillium: cercles concentriques dans certaines conditions.

Il se pose naturellement la question de savoir quel mécanisme intime pourrait bien présider à la génèse de ces formes curieuses. Lorsqu'on cherche dans la nature des formes et des dessins ressemblant à ceux qui nous intéressent, on est frappé par une analogie troublante : les colonies ressemblent aux dessins connus sous le
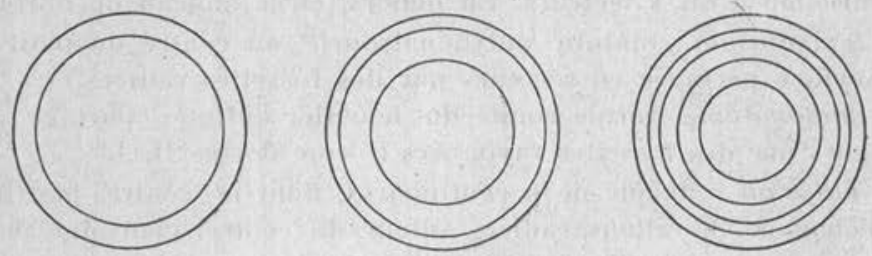

FIG. 4. - Vibrations circulaires d'un disque.
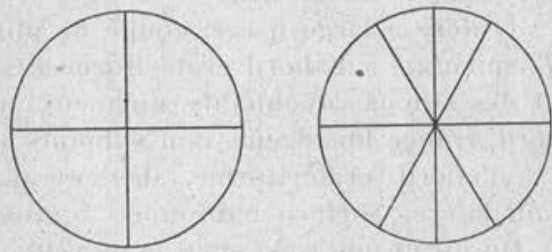

F1G. 5. - Vibrations radiaires d'un disque. Analogie avec Microsporum audouini.

nom de « figures de Chladni ». On se rappelle que Chladni a eu il y a 150 ans - le premier l'idée de mettre en évidence l'état vibratcire de plaques et de membranes, en versant du sable fin et en mettant ensuite ces plaques en vibrations à l'aide d'un archet. Le sable est chassé au niveau des ventres du mouvement ondulatoire pour s'accumuler aux lignes nodales qui restent ainsi marquées définitivement. Plus la forme de la plaque vibrante est régulière et l'oscillation provoquée simple, plus le dessin obtenu est, lui aussi, simple et régulier. On obtient ainsi, par des plaques rondes, les dessins suivants (fig. 4).

Avec l'augmentation du nombre des oscillations par seconde (c'est-à-dire de la fréquence), il se forme un nombre croissant de 
cercles concentriques, correspondant aux nœuds du mouvement vibratoire. Analogie : Trichophyton tonsurans, Trichophyton circuluscentrum.

La figure suivante (fig. 5) représente les oscillations radiaires d'une plaque circulaire. Ici, le nombre des diamètres augmente avec la fréquence.

Enfin, il existe des figures dites de combinaison, terme un peu inexact. Voici quelques exemples (fig. 6).

Ces figures de combinaison, très fréquentes dans les expériences de Chladni, naissent sous l'influence de petites asymétries des plaques et dépendent aussi de l'endroit où l'archet a mis la plaque en

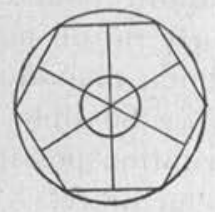

$\mathrm{H}$

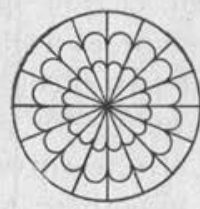

G

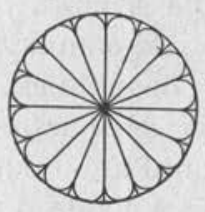

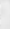

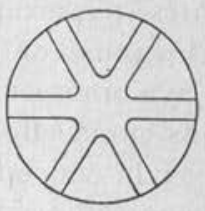

I

Fig. 6. - Figures de combinaison : G, Trichophyton rosaceum ; $\mathrm{H}$, Ctenomyces persicolor; I, Hemispora stellata.

vibration, ou des obstacles qui ont entravé le libre mouvement des plaques.

La similitude des figures de Chladni et des colonies serait-elle fortuite? On sait que, d'après le principe de Fournier, n'importe quelle figure géométrique peut être conçue comme le résultat de la superposition d'un nombre fini ou infini de courbes sinusoïdales d'amplitude et de longueur d'onde données. Le fait qu'une figure géométrique soit imitée par un mouvement vibratoire n'aurait done rien qui puisse surprendre. Seulement, ce serait vraiment une coïncidence assez surprenante que toutes les formes géométriques des colonies correspondent exactement à des états de vibration très simples ; s'il n'y avait pas de parenté plus profonde, on devrait s'attendre à trouver des exceptions plus fréquentes à ce parallélisme.

Etant donné que la forme géométrique d'un mouvement vibratoire reste la même dans tous les domaines de la physique et que les figures de Chladni ne sont qu'un moyen très commode pour étudier l'emplacement des nœuds et ventres d'un mouvement ondulatoire, tandis que les résultats obtenus gardent leur valeur générale par- 
tout, aussi bien en acoustique qu'en électricité ou ailleurs, nous nous croyons en droit d'admettre que les colonies des champignons naissent, elles aussi, sous l'influence de phénomènes rythmiques.

Quels sont les phénomènes biologiques rythmiques qui pourraient intervenir ? On songera d'abord à l'irritation et à l'onde d'irritation. Malheureusement, l'existence de la loi du « tout ou rien » et de la phase réfractaire font de l'onde d'irritation une entité à part, qui n'a de commun avec les vraies ondes que le nom. Ces deux lois empêchent la superposition de deux ondes à phase et direction opposées, ce qui serait nécessaire pour créer des * ondes stationnaires d'irritation ». Il est vrai que la validité de ces deux lois n'est prouvée jusqu'ici que pour la fibre nerveuse et pour les tiges de certaines plantes, par exemple Mimosa pudica, et que leur validité pour des entités plus complexes, comme la cellule et à plus forte raison pour des agglomérations de cellules, n'est pas encore établie ; mais nous allons nous efforcer de démontrer qu'il sera peut-être possible de sauver la conception d'une relation étroite entre l'irritation possible et la morphogénèse à l'aide d'une hypothèse supplémentaire.

Toutefois, nous ne prétendons nullement que l'irritation et ses particularités de rythme puissent être seules en cause. Nous discuterons plus tard la possibilité que d'autres phénomènes rythmiques interviennent. C'est seulement à cause d'une certaine probabilité que nous avons parlé de l'irritation en premier lieu. L'hypothèse supplémentaire, dont nous allons parler, reste applicable, mutatis mutandis, à tous les autres phénomènes rythmiques.

Quelle que soit done la nature du processus rythmique morphogénétique, nous nous posons la question de savoir s'il ne faut pas considérer à part, d'un côté le processus rythmique et d'autre part les moyens de sa propagation. Les phénomènes de l'irritation et la plupart, sinon tous les processus biologiques rythmiques, s'accompagnent de l'apparition de courants d'action locaux, et il se peut que ces courants locaux se chargent de la propagation, de la distribution et de la synchronisation des phénomènes rythmiques proprement dits. Quelques exemples rendront cette idée plus claire.

Des cellules de Nitella, en suspension dans une goutte d'eau, montrent, après quelques minutes, une parfaite synchronisation de leurs phénomènes rythmiques. On admet que des courants d'action, passant par l'eau de la goutte, se chargent de ce rôle de synchronisateurs. Autre exemple : les ondes céphaliques, enregistrées simultanément à partir des deux hémisphères, sont synchrones. Ce synchronisme disparaît après séparation des deux hémisphères par le couteau. Il réapparaît si l'on applique les deux faces du cerveau 
l'une sur l'autre. C'est la couche capillaire du liquide se trouvant entre les deux tranches qui s'est chargée de la transmission du courant d'action, indépendamment de toute structure vivante.

Il se peut donc que l'électricité soit le vecteur qui relie entre eux les divers éléments doués de rythmicité. Il se peut que l'irritabilité de la cellule soit le moteur de la rythmicité, mais que des phénomènes à part, c'est-à-dire les courants d'action locaux, se chargent des fonctions de propagation, de distribution et de synchronisation. Ces courants locaux, se dispersant essentiellemeent dans les espaces intercellulaires, formeraient, en se superposant, une sorte de champ électrique, qui serait done un champ d'excitation extracellulaire et non un champ d'irritation intracellulaire. Ces courants locaux sont

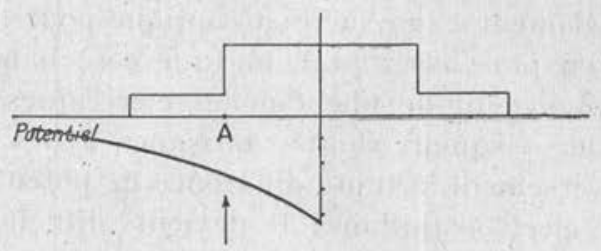

F16. 7. - Gradient du potentiel.

superposables, à l'encontre des courants d'action intercellulaires proprement dits, et indépendants, dans une certaine mesure, de l'ćtat d'irritation des fibres mycéliennes. La loi du « tout ou rien » n'existe pas pour ce « champ d'excitation morphogénétique ».

L'existence d'un champ d'excitation (et non d'irritation !) expliquerait facilement le fait que les tropismes des hyphes changent dans les colonies si souvent brusquement leur sens. Admettons que dans un cas donné l'intensité du courant d'excitation diminue du centre de la colonie vers la périphérie, d'après un gradient symbolisé dans le dessin suivant par un trait fort (fig. 7).

Au point A, l'intensité du potentiel tombe au-dessous du seuil de l'irritabilité. La loi du « tout ou rien », restant valable pour les hyphes, celles-ci ne sont plus excitées à partir de cet endroit, d'où changement dè la direction de leurs tropismes.

Nous avons déjà fait allusion à la possibilité qu'un autre phénomène rythmique que l'irritabilité puisse être à l'origine de la morphogénèse. Nous pensons en premier lieu aux intéressantes découvertes de Seifriz. Ce chercheur, travaillant sur des Myxomycètes, s'est posé la question suivante : Quelle est la pression hydraulique à l'intérieur de ce plasmode, et à quelle loi obéissent les change- 
ments de cette pression, qui est le moteur des courants protoplasmatiques dans les cellules?

La réponse obtenue était surprenante : la pression hydraulique à l'intérieur du plasmode change périodiquement. Ces changements de la pression sont d'une régularité absolue. En règle générale, on n'observe pas de simples rythmes sinusoïdaux, mais des courbes plus compliquées, dues à la superposition de plusieurs ondes de durée différente. Les courbes ubtenues sont différentes dans les différentes espèces de Myxomycètes et ont pour chaque espèce des caractères définis. Les changements de la pression observés étaient de l'ordre de grandeur de $10 \mathrm{~cm}$. d'eau. Seifriz a obtenu ces résultats par une méthode manométrique qui ne semble guère applicable à d'autres objets biologiques. Nous proposerons donc dans notre programme expérimental une méthode optique pour l'étude du phénumène. Il est en plus assez probable que ces changements de la pression s'accompagnent de phénomènes électriques. Tout mouvement d'un liquide visqueux donne naissance à des différences de potentiel, et, inversement, à toute différence de potentiel correspond un mouvement électro-capillaire. It devient difficile d'y séparer cause et effet.

Il reste évidemment possible que d'autres phénomènes rythmiques interviennent.

Mais, quelle que soit la nature du processus morphogénétique, quels que soient aussi les moyens de propagation du phénomène rythmique, il est clair pour nous que cette colonie ne peut plus être un simple amoncellement de fibres isolées, mais elle est, comme la plaque vibrante, un «tout ». L'emplacement des plis, cercles, sillons, etc... dépendra d'une série de constantes, dont une sera le diamètre de la colonie, en analogie complète avec la plaque vibrante.

Cette remarque nous ramène à cette question : Sommes-nous ici peut-être en présence d'un cas particulier d'un phénomène beaucoup plus général ? On cherche toujours, en embryologie animale, une définition pour ce " tout», pour mieux comprendre cette ordonnance et harmonie merveilleuse des parties. Un phénomène comme la métamérisation trouverait, par exemple, une explication toute simple en admettant que des processus rythmiques soient à son origine. Il y a de nombreuses analogies troublantes entre le comportement de corps vibrants et l'embryologie. Ainsi, pour ne citer qu'un exemple, a-t-on étudié ce qui advient à une figure de Chladni si l'on augmente peu à peu la fréquence des vibrations. Ces figures se transforment, dans un cas donné, d'après le dessin suivant (fig. 8 ). 
Des transformations tout à fait analogues se voient en embryologie. La formation de l'œil en donne un exemple. Si l'on compare le mécanisme de l’organogénèse de l'œil avec celui de la transformation des figures de Chladni, on ne peut qu'être frappé par ce parallélisme (fig. 9).

D'ailleurs, en embryologie, il est tout à fait superflu qu'un changement quelconque de la fréquence intervienne. Le simple fait de grandir rend l'embryon « trop grand 》 pour une fréquence donnée.
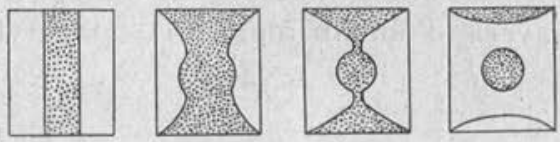

Fig. 8. - Déformation des figures de Chladni par augmentation successive de la fréquence des vibrations. Le pointillé facilite la comparaison avec la figure suivante.
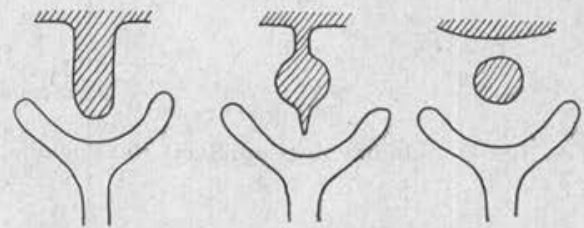

Fig. 9. - Organogénèse de l'œil d'un vertébré.

Tout se passe donc comme si un changement de la fréquence s'était produit, l'embryon gardant ses dimensions.

Programme expérimental. - Cette étude doit commencer par une analyse très poussée de l'histologie des cultures. Cette partie descriptive du travail devra être complétée par l'observation de la croissance des cultures à l'aide de marques colorées, à l'instar des méthodes de Vogt employées en embryologie animale.

Ce sont toujours les méthodes classiques de l'ẹbryologie qui doivent présider à une deuxième partie du travail, consacrée à l'étude de la croissance dans des conditions expérimentales. Il faudra examiner le comportement de morceaux de la colonie extirpés et transplantés pour éclaircir dans ce domaine les analogies possibles avec l'embryologie animale. C'est en particulier la question de la différenciation d'après le lieu de provenance ou celui de l'implantation 
qu'il s'agira d'étudier. (« Ortsgemässe und Herkunfts gemässe Differenzierung » de Speeman).

La question de la régénération doit être examinée.

Les expériences demanderont une technique de culture spéciale pour pouvoir faire les opérations nécessaires aussi bien du côté libre que du côté milieu nutritif.

Pour examiner l'influence de la forme et la taille de la culture (done l'influence du * tout $\gg$ ), on fera des cultures linéaires sur des fils et des cultures sur des supports de forme et taille différentes.

On étudiera dans la suite l'influence d'obstacles divers sur les cultures. On essayera d'obtenir des effets d'interférence à l'aide

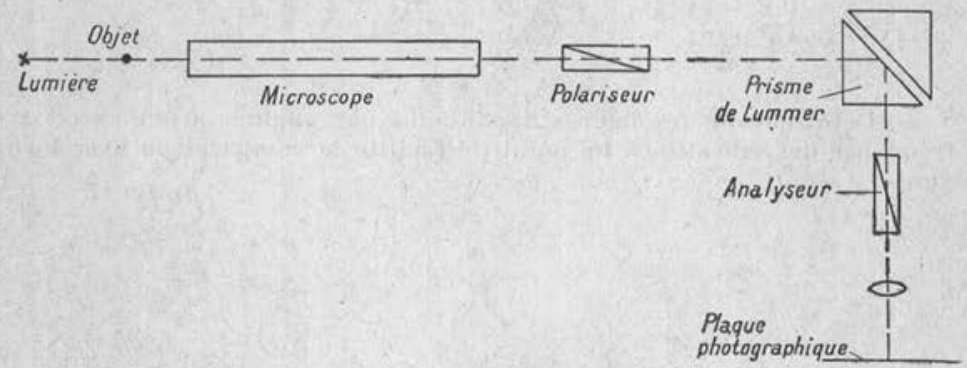

Fig. 10. - Schéma de l'appareil de Pockels.

d'obstacles appropriés pour obtenir des renseignements sur l'ordre de grandeur de la longueur d'onde de l'excitation morphogénétique, qui sera probablement d'un millimètre environ.

Quittant le domaine histologique, on examinera les phénomènes physiologiques ; en premier lieu, le comportement électrique, ceci au repos et après excitation traumatique, chimique ou électrique des colonies. On examinera l'électrotropisme des champignons soumis à des courants continus ou alternatifs d'intensité et de période données, ceci pour voir dans quelle mesure des phénomènes électriques sont à l'origine de la morphogénèse.

Il sera probablement impossible d'utiliser la méthode de Seifriz pour nos recherches. On pourrait peut-être y remédier par une méthode optique, dont voici les principes : les mouvements protoplasmiques sont à l'origine d'une double réfraction de Ia lumière. Ceci pour deux raisons: d'abord, parce que le mouvement d'un liquide visqueux donne naissance à la double réfraction par un phénomène d'alignement des molécules et des micelles, et, deuxièmement, parce que la matière vivante est constituée en partie par 
des cristaux liquides, qui, sous l'influence d'un changement de la pression extérieure, se déforment et donnent par là de nouveau naissance à une double réfraction.

Pour mettre en évidence cette double réfraction, on pourrait employer l'appareil de Pockels, dont voici le schéma (fig. 10). L'appareil est très sensible. Il décèle la présence de traces de lumière elliptiquement polarisée jusqu'à une différence de phases de $2 \pi / 2.000$, par le déplacement d'une fine bande d'interférence.

On pourrait essayer d'augmenter l'effet en plaçant l'objet dans un fort champ électrique ou magnétique. On crée ainsi une double réfraction artificielle supplémentaire, dont les changements, proportionnellement plus grands, seraient à étudier.

Institut de Parasitologie de la Faculté de Médecine de Paris (Directeur : Prof. E. Brumpt), Section de Mycologie (Chef de service : Dr M. Langeron). 